\title{
TO STUDY IMPACT OF BLOOD COMPONENT TRANSFUSION THERAPY IN ACUTE FEBRILE ILLNESS PATIENTS IN INTENSIVE CARE UNIT
}

\author{
Hedau Mayur Ramkrishnarao1, Nagar Vidya Sanjay²
}

${ }^{1}$ Assistant Professor, Department of Medicine, Grant Government Medical College, Mumbai.

2 Professor and HOD, Department of Medicine, Grant Government Medical College, Mumbai.

\section{ABSTRACT}

\section{BACKGROUND}

Acute Febrile Illness in an individual is defined as a fever of $38.3^{\circ} \mathrm{C}$ or more for at least 2 days or fever with no localising causes like pneumonia or diarrhoea (for the present study). Malaria, leptospirosis, dengue, typhoid fever and certain unidentified viral fevers are the usual causes in monsoon. The present study is planned to gain insight into the most common types of coagulation abnormalities seen in AFI and their effect on the course of disease.

\section{MATERIALS AND METHODS}

A descriptive study was performed in 100 indoor patients admitted in ICU with AFI with one or more of these coagulation abnormalities: Platelet count $<1,50,000 / \mathrm{mm}^{3}$, or patients with INR $>1.2$ or patients with PTTK prolonged by $>6$ seconds were included. Their clinical profile, biochemical parameters, severity of illness (SOFA Score), treatment received were correlated with outcome with Chi square test.

\section{RESULTS}

Out of these 100 cases, 56 were male with mean age of 26.46 years and 44 were female with mean age of 25.5 years, indicating that tropical diseases are more common in the working age group. Most of the patients suffered from Malaria (53), Leptospirosis (13), Dengue (7) in decreasing order whereas 27 had unidentified aetiology. Thrombocytopenia (100 \%), and prolonged bleeding time followed by INR (59\%) were most common deranged parameters. Mortality rates were high with patients having thrombocytopenia. ( $p=0.0281)$. Patients in ICU were studied according to SOFA Score mean (11.268) in survived and (>15) in expired patients.

\section{CONCLUSION}

1. The commonest coagulation abnormality in patients with AFI is Thrombocytopenia seen in almost all patients followed by raised INR (57), Prolonged PTTK (27), reduced serum fibrinogen levels. 2. Severe thrombocytopenia, raised INR, prolonged PTTK, reduced serum fibrinogen levels are poor prognostic markers of outcome in AFI patients. 3. Blood component therapy improved outcome in patients (90\%) who had clinically evident bleeding in presence of deranged coagulation parameters.

\section{KEYWORDS}

Acute Febrile Illness, Blood Component Therapy.

HOW TO CITE THIS ARTICLE: Ramkrishnarao HM, Sanjay NV. To study impact of blood component transfusion therapy in acute febrile illness patients in intensive care unit. J. Evolution Med. Dent. Sci. 2017;6(39):3156-3159, DOI: 10.14260/Jemds/2017/681

\section{BACKGROUND}

Fever is an elevation of body temperature that exceeds the normal daily variation and occurs in conjunction with an increase in the hypothalamic set point.(1,2) Fever is the most common symptom of presentation in a hospital with a spectrum of causes including infectious and non-infectious diseases that range from benign to life threatening.

A case of acute febrile illness (AFI) is defined for this study as any individual with fever for at least 2 days or temperature on admission of $38.3^{\circ} \mathrm{C}$ or greater with no identified localised cause of fever such as pneumonia, urinary tract infection (UTI), meningitis, diarrhoea, etc. $(1,2)$ Undifferentiated acute febrile illness (AFI) is a common clinical syndrome among patients seeking hospital care in this

Financial or Other, Competing Interest: None.

Submission 03-04-2017, Peer Review 04-05-2017,

Acceptance 10-05-2017, Published 15-05-2017.

Corresponding Author:

Dr. Hedau Mayur Ramkrishnarao,

Assistant Professor,

Department of Medicine,

Grant Government Medical College, Mumbai-88.

E-mail: drmayurhedau@gmail.com

DOI: $10.14260 /$ jemds $/ 2017 / 681$ institute. Such patients usually suffer from Malaria, Leptospirosis, Dengue, Typhoid fever, and certain unidentified viral fevers. Certain arthropod-borne infections are common in tropical regions because of favourable climatic conditions. Water-borne infections like leptospirosis are common due to contamination of water especially during the monsoon floods. Fever is the most common symptom of presentation in the hospital with a spectrum of causes including infectious and non-infectious diseases that range from benign and self-limiting to life threatening. Epidemiologically, common illnesses like malaria, dengue, and leptospirosis are very common in our setup and alteration of various haematological parameters have been observed in patients with the same. We aim to preview a clinical algorithm of acute cases of fever presenting with altered haematological parameters and the role of blood and blood component transfusion therapy on outcome and individual prognostic factor in various outcomes ranging from mortality to morbidity and the gamut of investigations required to obtain a diagnosis or a lack of it. Some of the febrile patients are critically ill and require treatment in Intensive Care Unit (ICU). Acute febrile illness patients constitute about $30-40 \%$ admissions in intensive care unit every year. These cases require institution of prompt specific 
therapy and in a few cases measures to normalize their haematological parameters. Sometimes to normalize these parameters these patients may require supportive therapy in the form of blood or blood component transfusion therapy, in addition to other supportive therapies for other organ dysfunction, such as dialysis, mechanical ventilation, inotropes, etc. Despite having multiple guidelines on indications of blood component therapy in individual derangements, there is no clear-cut evidence regarding benefit from such transfusions and in practice, transfusions also depend on the availability of blood and blood components. Hence, the present study is designed to evaluate impact of transfusions on outcome of the patient.

\section{Aims and objectives}

1. To evaluate the impact of blood and blood component therapy on outcome in critically ill patients of acute febrile illnesses in intensive care setup.

2. To compare the outcome in the form of development of organ failure, duration of ICU stay, need of organ support in patients with deranged haematological parameters receiving versus not receiving blood component therapy.

3. To study adverse effects of individual blood component therapy in such patients.

4. To determine the requirement of blood component therapy in patients with acute febrile illnesses.

\section{MATERIALS AND METHODS}

This was a descriptive study. 100 consecutive hospitalised patients admitted in ICU with diagnosis of acute febrile illnesses with deranged haematological parameters were included over a period of 1 year.

\section{Inclusion Criteria}

1. A case of acute febrile illness (AFI) with any individual with fever for at least 2 days and $<14$ days or temperature on admission of $38.3^{\circ} \mathrm{C}$ or greater with no identified localised cause of fever as pneumonia, urinary tract infection (UTI), meningitis, diarrhoea, etc.

2. Patient should have some haematological derangement either $\mathrm{Hb}<8 \mathrm{~g} \%$ or platelets $<1.2 \mathrm{lakh} / \mathrm{mm}^{3}$ or deranged coagulation profile.

3. Admission in MICU with MOD score of $>10$.

\section{Exclusion Criteria}

1. Patients with pre-existing haematological disorder like ITP, haemophilia.

2. Patients receiving blood component therapy prior to admission for same illness.

3. Patients who survive for less than $24 \mathrm{hrs}$. duration.

\section{Statistical Analysis}

Summary statistics were used for discrete variables. All categorically dependent and independent variables were assessed using the Chi square test or the Fisher's exact test. A p value of $<0.05$ at a $95 \%$ confidence level was considered statistically significant. Data was assessed in STATA SE 12.1.

\section{RESULTS}

Out of total patients screened over one year 100 patients were included in the study. Of these 12 patients were excluded of which 4 patients received blood component therapy prior to admission, 2 patients died within 24 hours and 6 had other infections like pneumonia, cellulitis and tuberculosis. So total 88 patients were studied in detail of which 57 were male and 31 were female, with age varying from 13 to 71 years with mean of 33.7 years (Table R1). Sixty-three patients were from Mumbai and 25 patients were outside Mumbai territory.

\begin{tabular}{|c|c|}
\hline No. of Cases & $\mathbf{8 8}$ \\
\hline Mean & 33.76 \\
SD & 14.28 \\
Range & $13-71$ years \\
\hline Gender & $57(64.8 \%)$ \\
Male & $31(35.2 \%)$ \\
Female & Table T1. Age and Sex Distribution of Patients \\
\hline \multicolumn{2}{|c|}{ Included in the Study } \\
\hline
\end{tabular}

\begin{tabular}{|c|c|c|}
\hline Aetiology & No. of Patients & Percentage \\
\hline Dengue & 06 & $06.8 \%$ \\
\hline $\begin{array}{c}\text { Hepatitis E with } \\
\text { Leptospirosis }\end{array}$ & 01 & $01.1 \%$ \\
\hline Leptospirosis & 15 & $17.1 \%$ \\
\hline Malaria & 25 & $28.4 \%$ \\
\hline Unidentified & 41 & $46.6 \%$ \\
\hline \multicolumn{2}{|c|}{ Table T2. Aetiology of Fever } \\
\hline
\end{tabular}

As Shown in Table T2 Most Common Aetiology being Unidentified Acute Febrile Illness followed by Malaria and Leptospirosis, Dengue in Decreasing Order-

- Malarial infections were diagnosed by peripheral smear and confirmed by OptiMAL malaria antigen test. Fourteen patients were positive for vivax malaria (6 were smear MP positive, 5 OptiMAL positive, and 3 positive by both). Eight patients were positive for falciparum malaria (2 smear MP positive, 4 OptiMAL positive, and 2 positive by both). Three were positive for mixed infection of both parasites (By either test).

- Dengue was diagnosed by NS1 antigen, IgM/IgG antibodies to Dengue or Dengue PCR. Two patients were Dengue PCR positive whereas 3 were IgM positive and 1 patient was NS1 antigen positive.

- Leptospirosis was diagnosed by Lepto Dri-Dot or ELISA (IgM antibodies to Leptospira) or Leptospira PCR when they presented in first 4 days. Seven patients were diagnosed by Leptospirosis PCR and 9 by Lepto Dri-Dot or IgM ELISA.

- Undiagnosed category included patients with acute fevers which were negative for malaria parasite on peripheral smear, negative for malarial antigen OptiMAL test, negative for dengue IgG/IgM antibodies, and Lepto Dri-Dot (IgM antibodies) negative. They didn't have any obvious focus of infection and were empirically treated with antimalarials and ceftriaxone. 


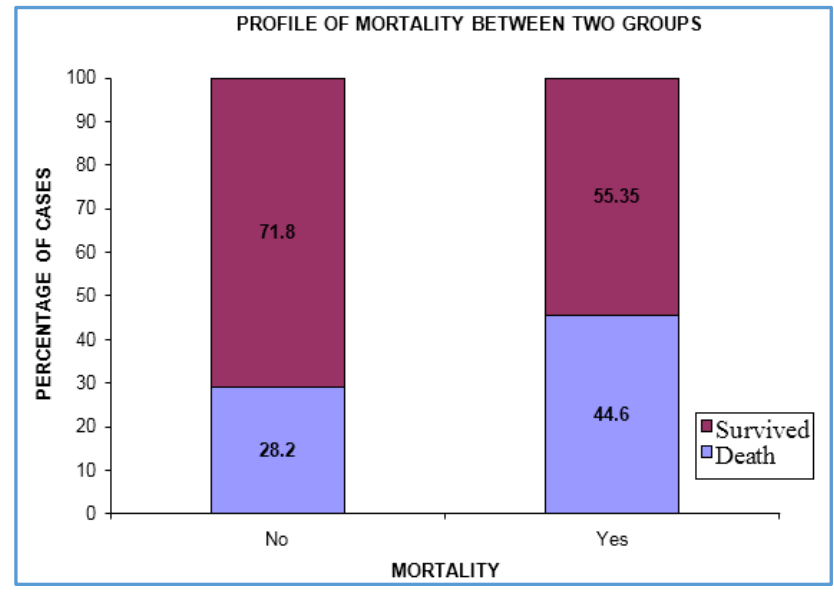

Figure F1. Comparison of Mortality Between Two Groups

By Chi Square Test $\mathrm{p}>0.05$ is Not Significant.

As per above figure, $71.8 \%$ of the patients who did not receive transfusions survived which was more than $55.35 \%$ of cases who received transfusion, but the difference was not significant.

\begin{tabular}{|c|c|c|c|}
\hline $\begin{array}{c}\text { Transfusion } \\
\text { Received }\end{array}$ & $\begin{array}{c}\text { Haemoglobin } \\
\text { n (\%) }\end{array}$ & $\begin{array}{c}\text { Death } \\
\text { n (\%) }\end{array}$ & $\begin{array}{c}\text { Survived } \\
\text { n (\%) }\end{array}$ \\
\hline $\begin{array}{c}\mathrm{No}(\mathrm{n}=32) \\
\mathrm{Hb} \mathrm{in} \mathrm{g} \% \\
\mathrm{Hb}<8\end{array}$ & $02(06.5)$ & $02(100)$ & -- \\
$\mathrm{Hb}>8$ & $30(93.75)$ & $07(24.1)$ & $22(75.9)$ \\
\hline $\mathrm{Yes}(\mathrm{n}=56)$ & $12(12.8)$ & $06(50.0)$ & $06(50.0)$ \\
$\mathrm{Hb}<8$ & $44(78.57)$ & $19(44.2)$ & $24(55.8)$ \\
$\mathrm{Hb}>8$ & Table T3. Profile of Transfusion with \\
\hline \multicolumn{3}{|c|}{ Haemoglobin and Mortality } \\
\hline
\end{tabular}

By Chi Square Test $*$ p $<0.05$ is Significant.

As per this study, $100 \%$ death rate was seen among patients who had $\mathrm{Hb}$ less than 8 and did not receive blood transfusion (whole blood/pack cells) which was significantly more than $24.1 \%$ death in patients who had $\mathrm{Hb}$ more than or equal to $8 \mathrm{~g} \%$ and did not receive transfusion of whole blood/pack cells.

This was statistically significant, $\mathrm{p}<0.05$. However, there is no significant difference in mortality in patients who received blood transfusion irrespective of $\mathrm{Hb}$. And mortality was higher in patients receiving blood/pack cell transfusion when $\mathrm{Hb}$ was $>8 \mathrm{~g} \%$ (44.2\% versus 24.1).

\begin{tabular}{|c|c|c|c|}
\hline $\begin{array}{c}\text { Transfusion } \\
\text { Received }\end{array}$ & $\begin{array}{c}\text { Platelet } \\
\text { n (\%) }\end{array}$ & $\begin{array}{c}\text { Death } \\
\text { n (\%) }\end{array}$ & $\begin{array}{c}\text { Survived } \\
\text { n (\%) }\end{array}$ \\
\hline No (n=32) & & & \\
Platelets/ $\mu \mathrm{L}$ & & & \\
Plt. $<50,000$ & $07(22.6)$ & $04(57.1)$ & $03(42.9)$ \\
Plt. 50,000-100,000 & $11(35.5)$ & $02(18.2)$ & $09(81.8)$ \\
Plt. $>100,000$ & $14(43.75)$ & $03(23.1)$ & $10(76.9)$ \\
\hline Yes (n=56) & $43(76.78)$ & $15(35.7)$ & $27(64.3)$ \\
Plt. $<50,000$ & $09(16.3)$ & $08(88.9)$ & $01(11.1)$ \\
Plt. 50,000-100,000 & $04(07.3)$ & $02(50.0)$ & $02(50.0)$ \\
Plt. > 100,000 & 02 Pable T4. Profile of Transfusion with \\
\hline \multicolumn{4}{|c|}{ Platelets and Mortality } \\
\hline \multicolumn{4}{|c|}{}
\end{tabular}

By Chi Square Test, $p<0.05$ is Significant
This study reveals that $57.1 \%$ cases died who had platelet count less than $50,000 / \mu \mathrm{L}$ and did not receive platelet transfusion, which was more than $18.2 \%$ mortality in cases having platelets in the range $50,000-100,000 / \mu \mathrm{L}$ and not receiving platelet transfusion. However, there was trend towards higher mortality in patients receiving platelet transfusion when platelet count was $>50,000 / \mu \mathrm{L}(88.9 \%$ Vs. $18.2 \%) \mathrm{p}<0.05$.

The mean platelet count of those who expired was $23,870 / \mu \mathrm{L}$, significantly lower than that of survived i.e. $32,971 / \mu \mathrm{L}$. It has also been shown in study by Gerardin et $\mathrm{al}^{(3)}$ in $\mathrm{P}$. falciparum patients. Those who received transfusion had significantly lower mean platelet count i.e. $21,044 / \mu \mathrm{L}$ than those who did not i.e. $36,890 / \mu \mathrm{L}$; as platelets were transfused in patients with clinical evidence of bleeding and prophylactically if platelet count was $<20,000 / \mu \mathrm{L}$. But platelet transfusion did not cause significant improvement in survival rate because survival was affected by multiple other factors such as delay in referral, other organ involvement, availability of supportive measures. Average platelet count was lowest in Leptospirosis patients i.e. 20,840/ $\mu \mathrm{L}$; highest being in patients with dengue fever i.e. $34,570 / \mu \mathrm{L}$. Average platelet count in malaria patients was $29,070 / \mu \mathrm{L}$; but there was no statistically significant difference among those values.

In our study, the INR varied from 0.9 to 5.5 with mean of 2.15. Of the patients with deranged INR $>2.5,15$ had mucosal bleeding and one had subdural haematoma. Total 27 patients received FFP.

\begin{tabular}{|c|c|c|c|}
\hline & Survived & Died & Total \\
\hline Transfused & $10(11.36 \%)$ & $17(19.31 \%)$ & $27(30.68 \%)$ \\
\hline Non-transfused & $30(34 \%)$ & $31(35.22 \%)$ & $61(69.31 \%)$ \\
\hline Total & $40(45.45 \%)$ & $48(54.54 \%)$ & $88(100 \%)$ \\
\hline Table T5. Profile of Transfusion with FFP in Patients with \\
Raised INR and Mortality \\
\hline
\end{tabular}

By Chi Square Test $\mathrm{p}>0.05$ is Not Significant

In our study, total 27 patients received FFP of which 17 died and 10 survived. In 61 patients not receiving FFP, 31 died and 30 survived but the difference was not statistically significant. Out of 15 patients having bleeding, 14 received FFP transfusion and nine died (mortality 64, 3\%). Eight out of 13 patients receiving FFP for only deranged parameter and no bleeding died (mortality 61.5\%). ( $p>0.05$ ).

Untoward effects of Transfusion: Out of 56 patients receiving either form of transfusions (blood/FFP/platelets), only 7 had some adverse effects and none were fatal. Four had rigors, two had extravascular haemolysis and one had TRALI (Transfusion-Related Acute Lung Injury) during transfusion that recovered within 24 hours.

In our study, total 42 patients required ventilator support, most of them had $\mathrm{Hb}<8 \mathrm{~g} \%$ and platelets $<50,000 / \mu \mathrm{L}$. Total 40 patients received inotropic support, most of them had deranged platelet count. There were 4 patients out of 8 with MOD score 3, and 3 out of 4 with MOD score 4. In our patients, total 12 patients required haemodialysis, 5 had acute febrile illness of unidentified aetiology followed by leptospirosis (3), malaria (3) and dengue (1). 


\section{DISCUSSION}

Tropical infections like Malaria, Dengue and Leptospirosis form a major bulk of admissions to various hospitals in Tropics; especially during the rainy season and have a huge impact on the health budget of the country like India. These infections though differ in their aetiology, have similar clinical presentation. Even though each of these tropical infections is known to have characteristic symptomatology, in clinical practice it is often impossible to distinguish between them on presentation. They also have similar laboratory parameters, with features like thrombocytopenia, liver dysfunction, renal failure, and hypotension.

A descriptive study was performed in 88 indoor patients admitted in ICU with acute fever with non-localising signs. Out of these, 57 were male and 31 were female with the mean age of 33.7 years. The Age distribution revealed that predominantly young people were affected, 54 out of 88 being in the age group of 15-35 years, indicating that tropical infections were more common in the working age group, thus highlighting the socioeconomic burden of these diseases as highlighted by Maniyar(4) in 2009. Most of the patients suffered from fever of unidentified aetiology (46\%) followed by malaria, leptospirosis, dengue in decreasing order as shown in table T2. Aetiology was sought for in all cases, all patients were investigated for malaria parasite on peripheral smear (thick and thin). They were subjected to malarial antigen test i.e. OptiMAL test which has a sensitivity equivalent to a trained technician looking at a thin blood film for thirty minutes. Because the test only detects live parasites, it can be used to monitor response to treatment. It has a sensitivity of $96.4 \%$ and specificity of $98.9 \%$.

Dengue fever was diagnosed by Dengue NS1 antigen in first 4 days and then by IgM antibodies to dengue virus. (Sensitivity being $98.9 \%$ and specificity being 97.9\%). Dengue PCR was also used as diagnostic tool in patients presenting early. Leptospirosis was diagnosed by Lepto DriDot (Dri-Dot is a new card agglutination test developed for the rapid diagnosis of leptospirosis. This is a screening test, which detects $\lg \mathrm{M}$ antibodies against Leptospira with a sensitivity of $90 \%$ and specificity of $92 \%$ ) or Leptospira PCR when patient presented in first 4 days.

Twenty-five patients (28\%) were positive for malaria on peripheral smear or by OptiMAL test. Six (6.8\%) patients were diagnosed to have Dengue out of which 2 patients had Dengue PCR positive and 3 had Dengue IgM antibodies positive and one by NS1 antigen detection. Sixteen (18.18\%) patients were diagnosed to have Leptospirosis, out of which 7 were PCR positive and 9 were Dri-Dot or IgM ELISA test positive.

Forty-one of these patients $(46.6 \%)$ were negative for available tests for malaria, dengue and leptospirosis. This can be probably explained by a few factors like since ours is a tertiary care centre, sizeable number of patients are referred after treatment and development of complications, and hence may have received antimalarials and antibiotics before presenting to us, thus clearing the parasitaemia. Leptospirosis and dengue antibody formation may take 10-14 days and patient may be discharged or may succumb prior to this. We relied on our clinical experience while awaiting laboratory analysis for treating patients and each of these patients received antimalarials (Artesunate + Doxycycline). Clindamycin was used in place of Doxycycline in pregnant patients and those with severely deranged LFTs. Antibiotics like ceftriaxone were added in patients suspected to have leptospirosis. Patients diagnosed with a localised site of infection (like pneumonia, liver abscess, upper or lower respiratory infection, urinary tract infection, pyogenic meningitis, etc.) were excluded from the study.

Out of 88 patients, 11 had petechial rash of which $5(45.45 \%)$ patients had Dengue fever, $4(36.36 \%)$ were undiagnosed AFI, 2(18.18\%) had Leptospirosis. All the patients with dengue had petechial rash, of which only 3 had who were diagnosed to have platelet count $<20,000 / \mu \mathrm{L}$; being one of the diagnostic criteria of Dengue haemorrhagic fever. However, in other patients who had petechial rash, all had platelet count $>20,000 / \mu \mathrm{L}$. Out of 88 patients, 62 were admitted to ICU with bleeding tendency ranging from mucosal bleed to alveolar haemorrhages and intra-cranial haemorrhage.

\section{CONCLUSION}

1. Transfusion of blood component therapy such as packed cell transfusion is most beneficial in improving outcome of patients who have upper GI haemorrhage. In patients without significant mucosal bleeding, there is no benefit of whole blood/packed cell transfusion where haemoglobin is more than $8 \mathrm{~g} \%$. Platelets transfusion is beneficial in patients with platelet count $<50,000 / \mu \mathrm{L}$ when associated with significant mucosal bleeding. There is a trend towards higher mortality in patients receiving platelet transfusion if baseline platelet count is $>50,000 / \mu \mathrm{L}$. Fresh frozen plasma transfusion has no significant impact on outcome in patients of acute febrile illness irrespective of INR, when patient has no significant clinical bleeding.

2. There is no significant impact of baseline $\mathrm{Hb}$ or platelet count on patient's duration of ICU stay. But transfusion of blood component therapy increases patient's duration of ICU stay.

3. There are no significant adverse effects of blood component therapy in critically ill patients of acute febrile illness that impact the outcome.

4. Platelets transfusion is required in patients who have significant thrombocytopenia with platelet count $<50,000 / \mu \mathrm{L}$ when associated with major bleeding like gastrointestinal, alveolar haemorrhages. Whole blood and packed cell transfusion is beneficial in patients with $\mathrm{Hb}<8 \mathrm{~g} \%$ and significant gastrointestinal bleeding, hypotension or significant cardiac compromise.

\section{REFERENCES}

[1] White NJ, Breman JG. Malaria. Harrison's principles of internal medicine. 17th edn. New York, McGraw-Hill 2008:1280-94.

[2] Dinarello CA, Porat R. Fever and hyperthermia. Harrison's principles of internal medicine. 17th edn. New York, McGraw-Hill 2008; p. 117.

[3] Gerardin P, Rogier C, Ka AS, et al. Prognostic value of thrombocytopenia in African children with falciparum malaria. Am J Trop Med Hyg 2002;66(6):686-91.

[4] Maniyar VP. Severe thrombocytopenia as an individual prognostic indicator of in a case of acute febrile illness-a study(dissertation). Maharashtra University of Health Sciences, Nashik. 2009; p. 32. 